\title{
Heart Substructures Delineation in Lung SBRT: Central and Ultra-Central Lesions
}

\author{
Rodríguez Plá M*, Dualde Beltrán D, Aliaga Chueca A, Soler Rodríguez M, García Gómez R, Jordá \\ Sorolla E, Maroñas Martín E and Ferrer Albiach E \\ Department of Radiation Oncology, Hospital Clinico Universitario de Valencia, Spain
}

*Corresponding author: Rodríguez Plá M, Department of Radiation Oncology, Hospital Clinico Universitario de Valencia, 46010

Valencia, Spain, Email: rodriguez_marpla@gva.es

\section{ARTICLE INFO}

Received: 畿 November 11, 2021

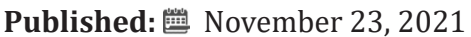

Citation: Rodríguez Plá M, Dualde Beltrán D, Aliaga Chueca A, Soler Rodríguez M, García Gómez R, et al., Heart Substructures Delineation in Lung SBRT: Central and Ultra-Central Lesions. Biomed J Sci \& Tech Res 40(1)-2021. BJSTR. MS.ID.006408.

Keywords: Heart Substructures; Lung SBRT; Central Lesions; Cardiac Toxicity

Abbreviations: RT: Radiotherapy; SBRT: Stereotactic Body Radiotherapy; BED: Biological Effective Dose; LMCA: Left Main Coronary Artery; LADCA: Left Anterior Descending Coronary Artery; RCA: Right Coronary Artery; RV: Right Ventricle; MACE: Major Adverse Cardiac Events

\begin{abstract}
Introduction: Stereotactic body radiation therapy (SBRT) remains a challenge in the treatment of central and ultra-central lung lesions. Intensity Modulated Radiation Therapy (IMRT) with image-guided radiation therapy (IGRT) minimizes toxicity to mediastinal organs such as the heart. This study aims to report the doses to the different substructures of the heart and the cardiac toxicity associated with SBRT treatment.

Materials/Methods: From 1 April 2018 to 31 December 2020, 28 patients with central lung lesions were treated with SBRT. The central lesions were defined according to RTOG 0813 criteria. Two fractions were used: 60Gy in 8 fractions or 50-55Gy in 5 fractions. Seventeen cardiac substructures were contoured following the atlas of Feng et al. and, subsequently, the dosimetry in these substructures and the cardiac toxicity of the patients were reviewed retrospectively according to CTCAE v5.0 grading. UK constraints were applied.
\end{abstract}

Results: Ten patients with early-stage non-small cell lung cancer, 15 metastatic lesions and 5 non-biopsied lesions suspected of PET-CT malignancy were treated. Mean follow-up was 16 months. Local control was $96 \%$ at 12 months. Cumulative probability of survival was $81 \%(95 \%$ CI $60-92 \%)$ at 12 months and $74 \%(95 \%$ CI 50-88\%) at 24 months. Six patients had died at the time of the analysis. No cancer-specific deaths were reported. There was no evidence of cardiac toxicity above grade 2 .

Conclusion: There were no deaths secondary to cardiovascular events with the doses received in the cardiac substructures. Constraints employed in the heart were safe. Prospective trials are necessary to elucidate possible cardiac toxicity taking each substructure of the heart into account.

\section{Introduction}

Stereotactic body radiation therapy (SBRT) has undergone exponential development in recent years, as their ablative capacity in tumour lesions. SBRT allows the administration of a high antitumor biologically effective dose (BED). Different fractions are used according to the anatomical location, size, and histology, among other factors. In general, the most frequent fractioning schemes used in SBRT are those in which a dose per fraction > 6 Gy is administered, in 1-5 fractions. Moreover, IMRT/VMAT with IGRT allows us to minimize toxicity to critical mediastinal organs as a heart [1,2]. In the Radiation Therapy Oncology Group (RTOG) 0617 study, higher cardiac doses were associated with worse overall survival (OS). This study observed that higher doses of thoracic radiation therapy were associated with more cardiovascular events [3]. Other authors have also reported the same findings [4,5]. The clinical limitations used in the treatment of SBRT, such as AAPM Task Group 101 or UK consensus, consider the heart as a single structure by limiting the dose to this organ $[6,7]$. Recently some 
publications try to elucidate whether the doses received by the different substructures of the heart impact on non-specific cancer mortality or increase cardiovascular events [8-11]. This study aims to report the doses to the different substructures of the heart and the cardiac toxicity associated with SBRT treatment in patients with central lung lesions treated in our centre.

\section{Materials and Methods}

From 1 April 2018 to 31 December 2020, 28 patients with 30 central lesions were treated with the SBRT technique with radical intent. Central and ultra-central lesions were defined according to RTOG 0813 criteria. Lesions located $2 \mathrm{~cm}$ from the mediastinum or main bronchus were defined as "central" [12]. Different immobilization systems (abdominal compression, vacuum cushion or head-neck-shoulders mask were used for treatment. ITV was defined by the 4D RPM-Varian ${ }^{\text {TM }}$ system. PTV was obtained by creating an isotropic (uniform) expansion of the ITV. Two fractions were used: 60Gy in 8 fractions or 50-55Gy in 5 fractions. UK consensus constraints were applied. For our study, 17 cardiac substructures were retrospectively contoured following the atlas of Feng, et al. [13] The atlas of Loap et al was also used for the contouring of the atrioventricular (AV) node [14]. Cardiac substructures were manually contoured by the same radiation oncologist (MRP) to avoid inter-observer variability. Contouring was performed in a CT without contrast. The following substructures were contoured: the cardiac chambers (right and left atrium, right and left ventricle), the superior and inferior vena cava, the pulmonary artery, the aortic arch, the ascending and descending aorta, the four cardiac valves (mitral, tricuspid, pulmonary and aortic), the left main coronary artery (LMCA), the left anterior descending coronary artery (LADCA), the circumflex artery, the right coronary artery (RCA) and the AV node. Subsequently, the dosimetry in these substructures and the cardiac toxicity of the patients were reviewed retrospectively according to CTCAE v5.0 grading.

\section{Results}

Ten patients with early-stage non-small cell lung cancer, 15 metastatic lesions and 5 non-biopsied lesions suspected of PETCT malignancy were analysed. Of these lesions, 26 were central and 4 ultra-central. The mean follow-up was 16 months (3-33). The mean age of the patients was 70 years (52-89). The mean GTV/PTV volume was 26/57.6cc (0.8-100/6.1-219). The baseline characteristics of the patients are described in Table 1.
Table 1: Patients and treatment characteristics.

\begin{tabular}{|c|c|}
\hline & n (\%) \\
\hline \multicolumn{2}{|c|}{ Age } \\
\hline Median & 70 \\
\hline Range & $52-89$ \\
\hline \multicolumn{2}{|c|}{ Sexo } \\
\hline Male & $21(75)$ \\
\hline Female & $7(25)$ \\
\hline \multicolumn{2}{|c|}{ Smoker } \\
\hline Yes & $22(78,5)$ \\
\hline No & $6(21,5)$ \\
\hline \multicolumn{2}{|c|}{ Prior cardiac history } \\
\hline Yes & $6(21,5)$ \\
\hline No & $22(78,5)$ \\
\hline \multicolumn{2}{|c|}{ Diabetes mellitus } \\
\hline Yes & $9(32)$ \\
\hline No & $19(68)$ \\
\hline \multicolumn{2}{|c|}{ Hypertension } \\
\hline Yes & $16(57,2)$ \\
\hline No & $12(42,8)$ \\
\hline \multicolumn{2}{|c|}{ Lesion type } \\
\hline Primary & $10(33,5)$ \\
\hline Metastases & $14(46,5)$ \\
\hline No biopsy & $6(20)$ \\
\hline \multicolumn{2}{|c|}{ Prescribed dose } \\
\hline 60 Gy in 8 fractions & $6(21,5)$ \\
\hline 55 Gy in 5 fractions & $7(25)$ \\
\hline 50 Gy in 5 fractions & $15(53,5)$ \\
\hline \multicolumn{2}{|c|}{ Treatment technique } \\
\hline Static beams (3DCRT or IMRT) & $0(0)$ \\
\hline VMAT & $28(100)$ \\
\hline
\end{tabular}

Once the substructures were contoured, the doses were reported at $0.5 \mathrm{cc}$ (D0.5cc) of each of them. D0.5cc and mean dose in heart were also reported (e.g. Figures $1 \& 2$ ). Table 2 and Table 3 shows the reported doses considering the two fractions used ( 5 and 8 fractions). In the 8 fractions group, median D0,5cc at each substructure was: right and left atrium, 7.5Gy(1-20) and 17.2Gy (1-48); right and left ventricle, 6.5Gy(1-12) and 3.5Gy (117); superior vena cava, 48Gy (6.8-52.8); inferior vena cava, $1 \mathrm{~Gy}$ (0-2); pulmonary artery, 17Gy (1-43); ascending and descending aorta,17.1Gy (1-39) and 18.8Gy(1-54.8); aortic valve, 2Gy (0-19.5); 
pulmonic valve, 4.5Gy(0-10); mitral valve, 0.5Gy (0-14); tricuspid valve, 0.5Gy (0-13.5); left main coronary artery, 7Gy (0-13.8); left anterior descending artery, 5Gy (0-15.6); left circumflex, 3Gy (0-
18.5); right coronary artery, 2.5Gy (0-10) and AV node,1.5Gy (112.6). Median D0.5cc and mean dose in heart were 47Gy (1-51.5) and 2.9 Gy $(0.1-10.8)$ respectively.

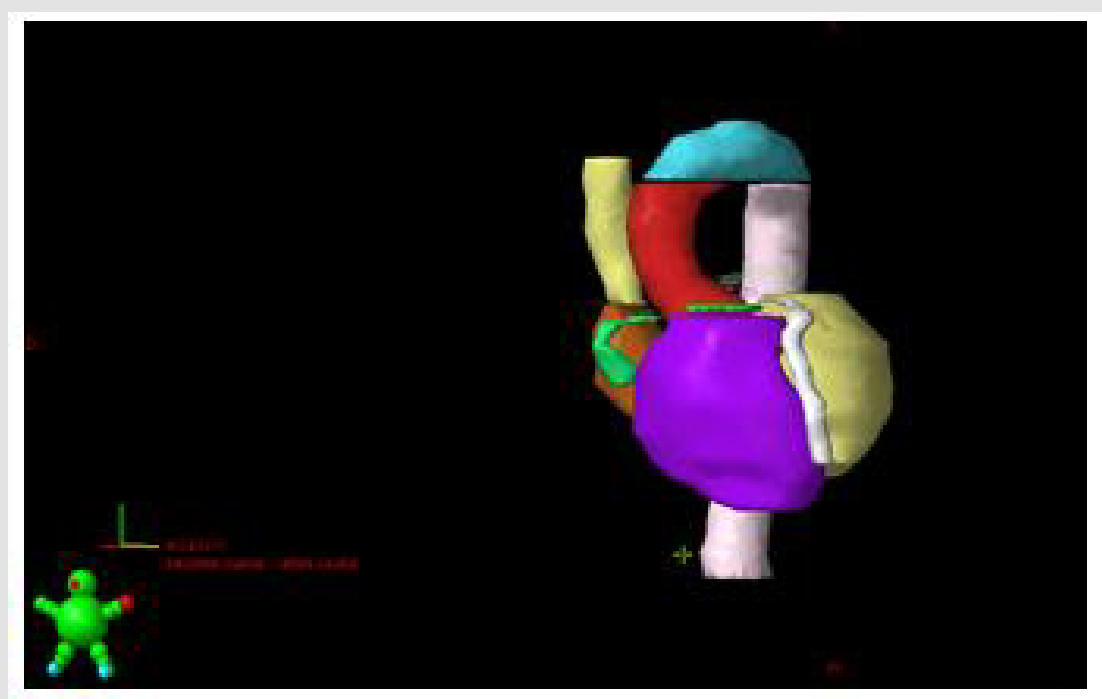

Figure 1: Contouring of cardiac substructures.
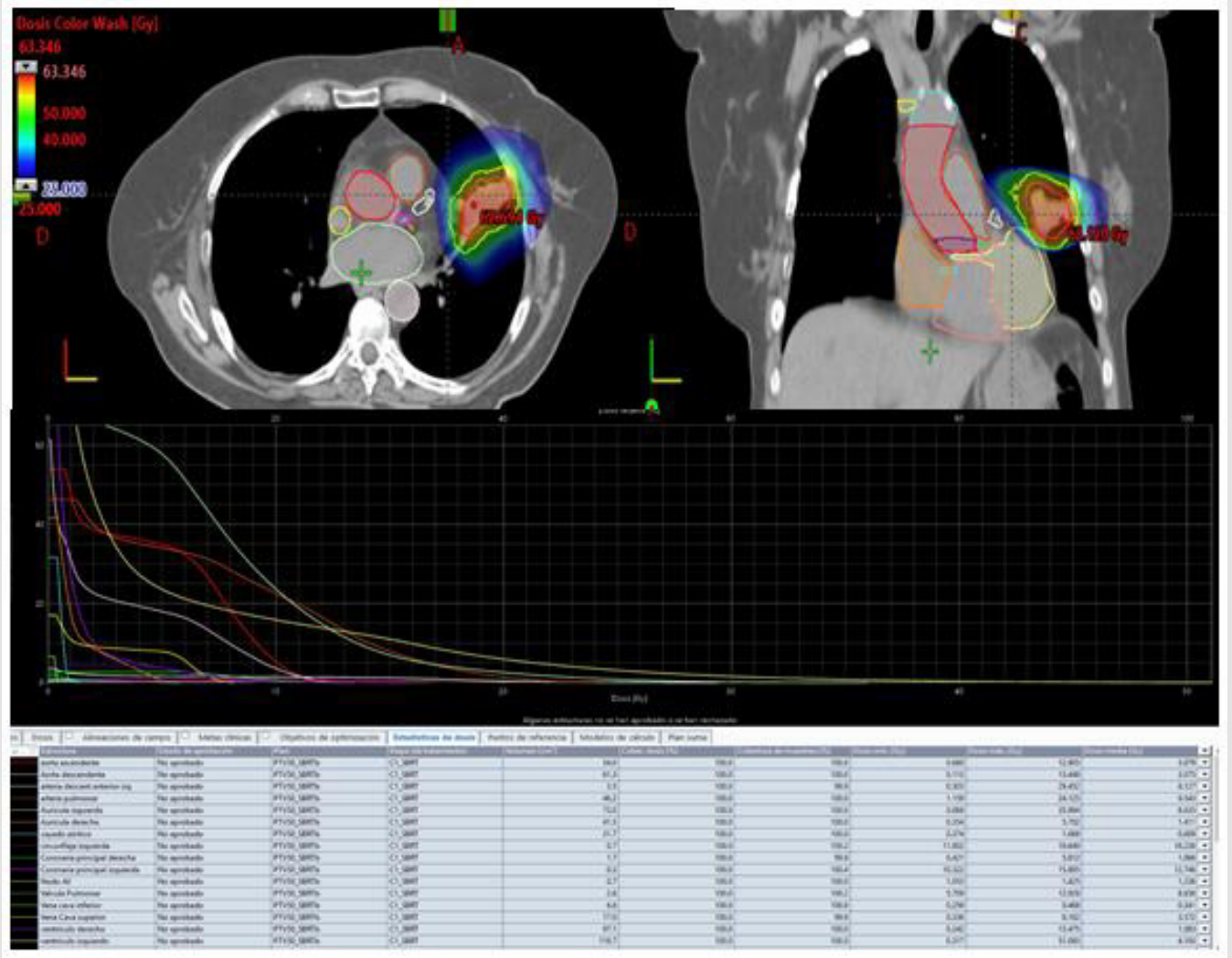

Figure 2: 67-year-old patient with personal history of resected lung adenocarcinoma. Patient had lung distant relapse. We administrated 50Gy in 5 fractions (10Gy/fraction). Dosimetry and dose-volume histogram (DVH) are showed. 
Table 2: Summary of reported doses.

\begin{tabular}{|c|c|c|c|c|}
\hline \multicolumn{5}{|c|}{8 fractions group median D0,5 cc } \\
\hline Substructure & Dose (Gy) & Range (Gy) & EQD2 (Gy) & EQD2 Range (Gy) \\
\hline Right atrium & 7.5 & $1-20$ & 5,51 & $0,53-22,5$ \\
\hline Left atrium & 17,2 & $1-48$ & 17,85 & $0,53-96$ \\
\hline Right ventricle & 6.5 & $1-12$ & 4,57 & $0,53-10,5$ \\
\hline Left ventricle & 3.5 & $1-17$ & 2,13 & $0,53-17,53$ \\
\hline Superior vena cava & 48 & $6.8-52.8$ & 96,00 & $4,85-113,52$ \\
\hline Inferior vena cava & 1 & $0-2$ & 0,53 & $0-1,13$ \\
\hline Pulmonary artery & 17 & $1-43$ & 17,53 & $0,53-79,28$ \\
\hline Ascending aorta & 17.1 & $1-39$ & 17,69 & $0,53-67,03$ \\
\hline Descending aorta & 18.8 & $1-54.8$ & 20,45 & $0,53-121,23$ \\
\hline Aortic valve & 2 & $0-19.5$ & 1,13 & $0-21,63$ \\
\hline Pulmonic valve & 4.5 & $0-10$ & 2,88 & $0-8,13$ \\
\hline Mitral valve & 0.5 & $0-14$ & 0,26 & $0-13,13$ \\
\hline Tricuspid valve & 0.5 & $0-13.5$ & 0,26 & $0-12,45$ \\
\hline Left main coronary artery & 7 & $0-13.8$ & 5,03 & $0-12,85$ \\
\hline $\begin{array}{l}\text { Left anterior descending } \\
\text { artery }\end{array}$ & 5 & $0-15.6$ & 3,28 & $0-15,41$ \\
\hline Left circumflex & 3 & $0-18.5$ & 1,78 & $0-19,95$ \\
\hline Right coronary artery & 2.5 & $0-10$ & 1,45 & $0-8,13$ \\
\hline AV node & 1.5 & $1-12.6$ & 0,82 & $0,53-11,26$ \\
\hline Median D0,5cc heart Dose & 47 & $1-51.5$ & 92,53 & $0,53-108,63$ \\
\hline Mean Heart Dose & 2.9 & $0.1-10.8$ & 1,71 & $0,05-9,05$ \\
\hline
\end{tabular}

In the 5 fractions group, median D0.5cc at each substructure was: right and left atrium, 3Gy (1-39) and 5Gy (1-31.5); right and left ventricle, 1.5Gy (1-14.3) and 3Gy (1-37.5); superior vena cava, 12Gy (1.7-53.1); inferior vena cava, 0.2Gy (0-13.2); pulmonary artery,13.75Gy (0.7-28.8); ascending and descending aorta, 12.2Gy (5-33) and 13.3Gy (5.9-49.6); aortic valve, 0.5Gy (0-15.5); pulmonic valve, $1.5 \mathrm{~Gy}$ (0.3-13); mitral valve, 0.5Gy (0-11); tricuspid valve, 0.4Gy (0-12); left main coronary artery, 0.9Gy (0.1-10); left anterior descending artery, 0.6Gy (0.1-8.8); left circumflex, 0.6Gy (0.1-15.7); right coronary artery, $0.5 \mathrm{~Gy}(0-12.8)$ and $\mathrm{AV}$ node, $0.4 \mathrm{~Gy}(0.2-9.8)$. Median D0.5cc and heart mean dose were 16Gy (0.8-43.1) and $1.1 \mathrm{~Gy}(0.1-10)$ respectively. Local control was $96 \%$ at 12 months.

Table 3: Summary of reported doses.
Cumulative probability of survival was $81 \%$ (95\% CI 60-92\%) at 12 months and $74 \%$ (95\% CI 50-88\%) at 24 months (Figure 3). On the other hand, post-treatment cardiovascular events were collected, especially major adverse cardiovascular events (MACE), including unstable angina, heart failure, myocardial infarction, and mortality secondary to a cardiac event. This data were collected through the patient's electronic history. Patients were followed up every 3-4 months for the first two years and every 6 months thereafter. With a mean follow-up of 16 months, no MACE was observed. Six patients had died at the time of the analysis. No major grade 2 cardiac toxicities were reported according to CTCAE v5.0 grading (Table 3).

\begin{tabular}{|c|c|c|c|c|}
\hline \multicolumn{5}{|c|}{$\mathbf{5}$ fractions group median D0,5 cc } \\
\hline Substructure & Dose (Gy) & Range (Gy) & EQD2 (Gy) & EQD2 Range (Gy) \\
\hline Right atrium & 3 & $1-39$ & 1,95 & $0,55-95,55$ \\
\hline Left atrium & 5 & $1-31,5$ & 3,75 & $0,55-65,36$ \\
\hline Right ventricle & 1,5 & $1-14,3$ & 0,86 & $0,55-17,37$ \\
\hline Left ventricle & 3 & $1-37,5$ & 1,95 & $0,55-89,06$ \\
\hline Superior vena cava & 12 & $1,7-53,1$ & 13,20 & $0,99-167,53$ \\
\hline Inferior vena cava & 0,2 & $0-13,2$ & 0,10 & $0,00-15,31$ \\
\hline Pulmonary artery & 13,75 & $0,7-28,8$ & 16,33 & $0,37-55,87$ \\
\hline
\end{tabular}




\begin{tabular}{|c|c|c|c|c|}
\hline Ascending aorta & 12,2 & $5-33$ & 13,54 & $3,75-70,95$ \\
\hline Descending aorta & 13,3 & $5,9-49,6$ & 15,49 & $4,69-147,81$ \\
\hline Aortic valve & 0,5 & $0-15,5$ & 0,26 & $0,00-19,76$ \\
\hline Pulmonic valve & 1,5 & $0,3-13$ & 0,86 & $0,15-14,95$ \\
\hline Mitral valve & 0,5 & $0-11$ & 0,26 & $0,00-11,55$ \\
\hline Tricuspid valve & 0,4 & $0-12$ & 0,21 & $0,00-13,20$ \\
\hline Left main coronary artery & 0,9 & $0,1-10$ & 0,49 & $0,05-10,00$ \\
\hline $\begin{array}{l}\text { Left anterior descending } \\
\text { artery }\end{array}$ & 0,6 & $0,1-8,8$ & 0,32 & $0,05-8,27$ \\
\hline Left circumflex & 0,6 & $0,1-15,7$ & 0,32 & $0,05-20,17$ \\
\hline Right coronary artery & 0,5 & $0-12,8$ & 0,26 & $0,00-14,59$ \\
\hline AV node & 0,4 & $0,2-9,8$ & 0,21 & $0,10-9,70$ \\
\hline Median D0,5cc heart Dose & 16 & $0,8-43,1$ & 20,80 & $0,43-114,43$ \\
\hline Mean Heart Dose & 1,1 & $0,1-10$ & 0,61 & $0,05-10,00$ \\
\hline
\end{tabular}

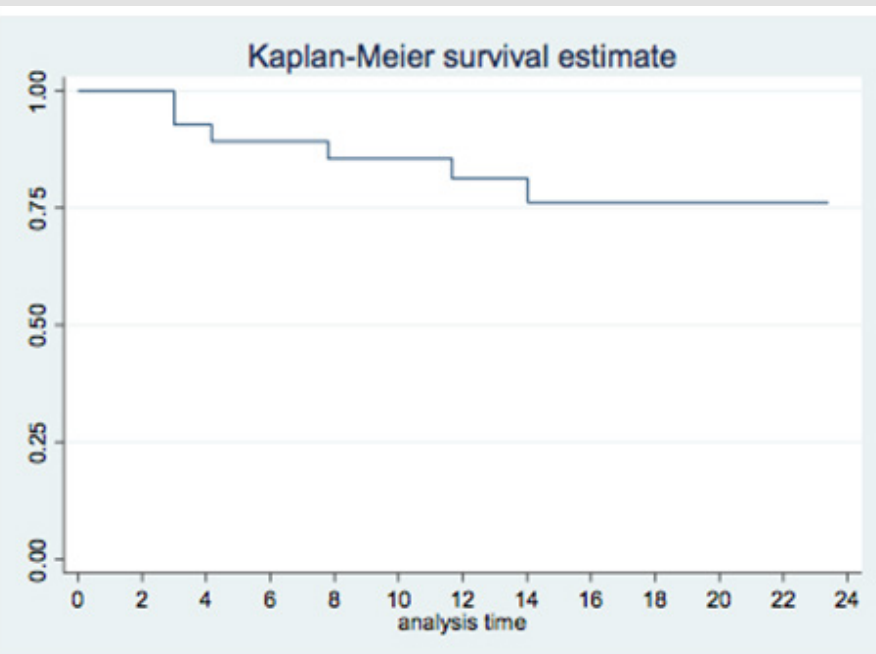

Figure 3: Kaplan-Meier curves and estimates of survival data.

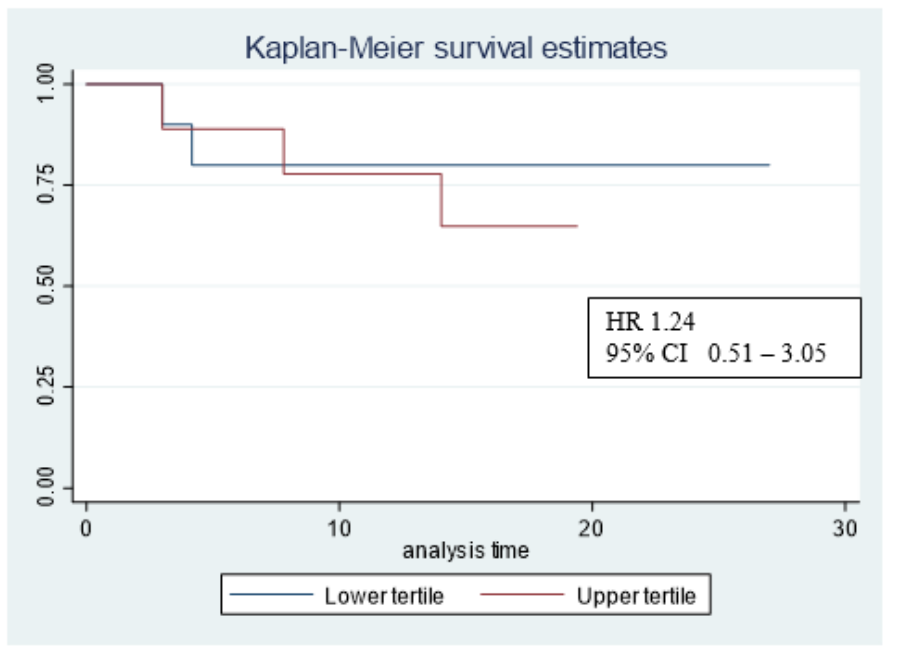

Figure 4: Kaplar-Meier graph corresponding the upper and lower bilateral ventricles max dose tertiles. 


\section{Discussion}

Different atlases have been developed for several disease sites in order to standardize target delineation. It is known that organat-risk delineation is also important. The heart, along with the main bronchus, is one of the main risk organs to consider in pulmonary SBRT of central and ultra-central tumours. For this reason, different studies have aimed to standardize contouring this organ and analyse the possible cardiac toxicity associated with this treatment [8-11]. When analysing the results obtained in the studies, we must take into account that we found different atlases for contouring cardiac substructures. Feng et al. describe the delimitation of 17 cardiac structures on CT with and without contrast. This group divides the heart into the following structures: 4 cardiac chambers $(2$ atriums, 2 ventricles), blood vessels (ascending and descending aorta, LADCA, LMCA, RCA, left circumflex), superior and inferior vena cava, 4 cardiac valves (mitral, tricuspid, pulmonary and aortic) and the AV node [13]. Duane et al. detail the delimitation of 15 cardiac structures employing CT without contrast. This study defines 5 segments in the left ventricle (anterior, lateral, apical, septal and inferior) and 10 segments in the coronary arteries (LMCA, LADCA: proximal, mid and distal; circumflex coronary artery: proximal and distal; RCA: proximal, mid, distal and posterior descending).

Both, Feng et al. and Duane et al., conclude that the use of an atlas for contouring cardiac substructures improves the precision in their delimitation. Likewise, Feng et al. reported that there were no significant differences in the variability of the contour of the substructures when using CT with or without contrast. On the other hand, Duane et al. report greater inter-observer differences in the contour of the segments of the coronary arteries than in the contour of the segments of the left ventricle [15]. Following this line, Danish cancer group recently published an article that attempts to clarify inter-observer variations in the contour of cardiac substructures [16]. This group segmented the heart and coronary arteries according to the atlas of Duane et al. with the difference that they divide the left ventricle into 4 segments (anterior, inferior, septal and lateral). This group also concluded that inter-observer differences are greater in small-volume structures such as the subsegments of coronary arteries. Other authors have also reported the same findings $[13,15]$.

In our study, the atlas of Feng et al. was used for two main reasons: to reduce inter-observer variability associated with the segmentation of small volumes (such as the subsegmentation of coronary arteries) and to report the doses of the 4 cardiac valves due to their possible involvement in cardiovascular events. The growing interest in knowing the possible cardiac toxicity associated with radiation therapy treatment has resulted in the development of software that allows for automatic segmentation of the heart (self- segmentation/auto-contouring). In some studies this software has already been used to contour cardiac substructures $[8,10]$. Some authors already report data on its use. For example, Farrugia $\mathrm{M}$ et al. conclude that small volumes, such as coronary arteries, require manual contouring since auto-contouring does not present an adequate anatomical correlation [17]. However, other authors such as Zhou $\mathrm{R}$ et al. conclude that self-segmentation of most cardiac substructures was at least comparable to manual delimitation [18]. On the other hand, focusing on cardiac toxicity reported to SBRT treatment, we must consider that published studies differ in dosimetric parameters studied and in cardiac atlases used. Reshko $\mathrm{L}$, et al. retrospectively analyse the dose at $0.3 \mathrm{cc}$ (D0.3cc) and the mean dose to cardiac substructures in 74 patients treated with lung SBRT. Delimitation of the substructures was carried out using selfsegmentation software. Reshko L et al. reported cardiovascular (CV) events in 18 patients ( $25 \%$ with a toxicity > G2), but this toxicity was not associated with the cardiac dose received [8]. However, an increase in cardiovascular events was observed in patients with a previous cardiac history.

In our study, 6 of the patients had a cardiovascular history but no increase in cardiovascular events after treatment was observed in these patients. Further follow-up of our patients is necessary, as Reshko et al. reported the occurrence of cardiovascular events 19 months after the end of treatment. On the other hand, other authors do find correlation with the cardiac dose received and non-cancerrelated death. Chan et al. correlated the dose to the right ventricle as a negative predictor of overall survival. This group observed that patients who had a V10Gy $<4 \%$ in the right ventricle had a higher overall survival than those with V10Gy $>4 \%$ [11]. In our study, 4 patients had a V10Gy $>4 \%$ in the right ventricle. Two of these patients had died at the time of analysis, both from a lung infection. Stam B et al. evidenced that the maximum dose received in the left atrium was associated with non-cancer-specific death in multivariate analysis (MVA) $(\mathrm{p}<0.05)$ [10]. However, Wong et al. observed that a higher dose in the ventricles correlated with an increase in non-cancer-specific death in the MVA $(\mathrm{p}<0.05)$ [9]. In our study, we compared non-cancer-related deaths in the upper and lower bilateral ventricles max dose tertiles (Figure 4). Limited sample size does not allow us to analyse this findings. The main limitations of our study are its retrospective nature and the number of patients included. This number of patients is due to the fact that, unlike other published studies, only patients with central lesions (and not all patients treated with lung SBRT) have been selected. Another limitation is the heterogeneity in the type of lesions treated (primary and metastatic lesions). These limitations prevent the extraction of robust conclusions and only allow a descriptive analysis of the patients treated in our centre. 


\section{Conclusion}

There were no deaths secondary to cardiovascular events with the doses received in the cardiac substructures Constraints employed in the heart were safe. The contouring of the cardiac substructures do not have a routine clinical task. Furthermore, no consensus for each heart substructure constraints has been met yet. Knowing the dose that each substructure of the heart can tolerate may be useful for the treatment of central and ultra-central lesions in certain clinical situations (e.g. multiple lesions or re-irradiation). Prospective trials and long follow-up are necessary to elucidate possible cardiac toxicity taking each substructure of the heart into account.

\section{Conflicts of Interest}

The authors declare no conflict of interest.

\section{References}

1. Chun SG, Hu C, Choy H, Komaki RU, Timmerman RD, et al. (2017) Impact of Intensity-Modulated Radiation Therapy Technique for Locally Advanced Non-Small-Cell Lung Cancer: A Secondary Analysis of the NRG Oncology RTOG 0617 Randomized Clinical Trial. J Clin Oncol 35(1): 5662.

2. Kole TP, Aghayere O, Kwah J, Yorke ED, Goodman KA (2012) Comparison of heart and coronary artery doses associated with intensity-modulated radiotherapy versus three-dimensional conformal radiotherapy for distal esophageal cancer. Int J Radiat Oncol Biol Phys 83(5): 1580-1586.

3. Bradley JD, Paulus R, Komaki R, Masters G, Blumenschein G, et al. (2015) Standard-dose versus high-dose conformal radiotherapy with concurrent and consolidation carboplatin plus paclitaxel with or without cetuximab for patients with stage IIIA or IIIB non-small-cell lung cancer (RTOG 0617): A randomised, two-by-two factorial phase 3 study. Lancet Oncol 16(2): 187-199.

4. McWilliam A, Kennedy J, Hodgson C, Vasquez Osorio E, Faivre-Finn C, et al. (2017) Radiation dose to heart base linked with poorer survival in lung cancer patients. Eur J Cancer 85: 106-113.

5. Gagliardi G, Constine LS, Moiseenko V, Correa C, Pierce LJ, et al. (2010) Radiation dose-volume effects in the heart. Int J Radiat Oncol Biol Phys 76(3): S77-S85.

6. Benedict SH, Yenice KM, Followill D, Galvin JM, Hinson W, et al. (2010) Stereotactic body radiation therapy: the report of AAPM Task Group 101. Med Phys 37(8): 4078-4101.

\section{ISSN: 2574-1241}

DOI: 10.26717/BJSTR.2021.40.006408

Rodríguez Plá M. Biomed J Sci \& Tech Res

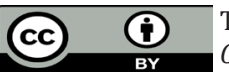

This work is licensed under Creative Commons Attribution 4.0 License

Submission Link: https://biomedres.us/submit-manuscript.php
7. Hanna GG, Murray L, Patel R, Jain S, Aitken KL, et al. (2018) UK Consensus on Normal Tissue Dose Constraints for Stereotactic Radiotherapy. Clin Oncol (R Coll Radiol) 30(1): 5-14.

8. Reshko LB, Kalman NS, Hugo GD, Weiss E (2018) Cardiac radiation dose distribution, cardiac events and mortality in early-stage lung cancer treated with stereotactic body radiation therapy (SBRT). J Thorac Dis 10(4): 2346-2356.

9. Wong OY, Yau V, Kang J, Glick D, Lindsay P, et al. (2018) Survival Impact of Cardiac Dose Following Lung Stereotactic Body Radiotherapy. Clin Lung Cancer 19(2): e241-e246.

10. Stam B, Peulen H, Guckenberger M, Mantel F, Hope A, et al. (2017) Dose to heart substructures is associated with non-cancer death after SBRT in stage I-II NSCLC patients. Radiother Oncol 123(3): 370-375.

11. Chan ST, Ruan D, Shaverdian N, Raghavan G, Cao M, et al. (2020) Effect of Radiation Doses to the Heart on Survival for Stereotactic Ablative Radiotherapy for Early-stage Non-Small-cell Lung Cancer: An Artificial Neural Network Approach. Clin Lung Cancer 21(2): 136-144.e1.

12. Bezjak A, Paulus R, Gaspar LE, Timmerman RD, Straube WL, et al. (2019) Safety and Efficacy of a Five-Fraction Stereotactic Body Radiotherapy Schedule for Centrally Located Non-Small-Cell Lung Cancer: NRG Oncology/RTOG 0813 Trial. J Clin Oncol 37(15): 1316-1325.

13. Feng M, Moran JM, Koelling T, Chughtai A, Chan JL, et al. (2011) Development and validation of a heart atlas to study cardiac exposure to radiation following treatment for breast cancer. Int J Radiat Oncol Biol Phys 79(1): 10-18.

14. Loap P, Servois V, Dhonneur G, Kirov K, Fourquet A, et al. (2021) A Radiation Therapy Contouring Atlas for Cardiac Conduction Node Delineation. Pract Radiat Oncol 11(4): e434-e437.

15. Duane F, Aznar MC, Bartlett F, Cutter DJ, Darby SC, et al. (2017) A cardiac contouring atlas for radiotherapy. Radiother Oncol 122(3): 416-422.

16. Milo MLH, Offersen BV, Bechmann T, Diederichsen ACP, Hansen CR, et al. (2020) Delineation of whole heart and substructures in thoracic radiation therapy: National guidelines and contouring atlas by the Danish Multidisciplinary Cancer Groups. Radiother Oncol 150: 121-127.

17. Farrugia M, Yu H, Singh AK, Malhotra H (2021) Autosegmentation of cardiac substructures in respiratory-gated, non-contrasted computed tomography images. World J Clin Oncol 12(2): 95-102.

18. Zhou R, Liao Z, Pan T, Milgrom SA, Pinnix CC, et al. (2017) Cardiac atlas development and validation for automatic segmentation of cardiac substructures. Radiother Oncol 122(1): 66-71.

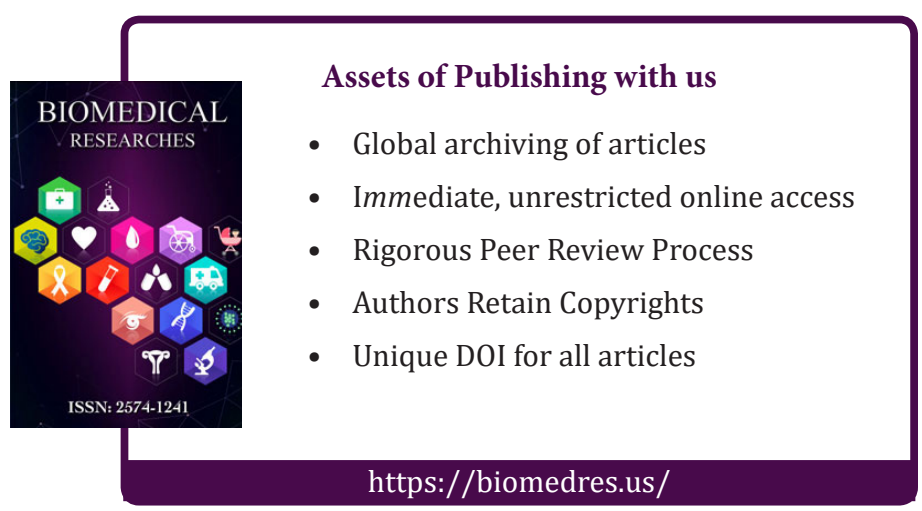

